\title{
Smart Homecare System for Health Tele-monitoring
}

\author{
Peter Leijdekkers, Valérie Gay, Elaine Lawrence \\ Faculty of IT, University of Technology Sydney \\ UTS FIT, PO box 123, Broadway 2007 NSW Australia \\ E-mail: [peterl, valerie, elaine]@it.uts.edu.au
}

\begin{abstract}
An increasing aged population worldwide puts our medical capabilities to the test. Research and commercial groups are investigating novel ways to care for the aged and chronically ill both in their own homes and in care facilities. This paper describes a prototype we have developed for remote healthcare monitoring. This personalized smart homecare system uses smart phones, wireless sensors, web servers and IP webcams. To illustrate the functionality of the prototype we describe a series of typical tele-health monitoring scenarios.
\end{abstract}

\section{Keywords-component; smart phones, Bluetooth, telehealth}

\section{INTRODUCTION}

The aged population has been increasing dramatically worldwide. The global population of people over the age of 65 is expected to more than double from 375 million in 1990 to 761 million by 2025 [1]. Coping with a disproportionate aging population presents an enormous healthcare problem for national governments and society. The financial and staffing cost of caring for the escalating numbers of aged persons in nursing homes or hospitals will be a huge challenge. For those seniors who prefer to live independently in their own homes, there is an increased risk of falls and strokes which could prove life threatening. In fact, 'in 2002, nearly 13,000 people ages 65 and older died because of fall-related injuries' [2]. Statistically, more than $60 \%$ of people who die from falls are 75 and older [3]. The New England Journal of Medicine states that the chances of surviving a fall, heart attack or stroke are six times greater if the senior gets help within an hour [4]. Gururajan et al [5] stated most healthcare information is both time and life critical, so it must be captured and/or delivered whenever and wherever needed. Our smart homecare system provides this capability with the added benefit to users that they may stay in their own homes, comforted by the knowledge that their conditions are being as closely monitored as if they had a personal health caregiver in their home.

Healthcare providers need to devise cheaper and more novel ways to manage and care for sufferers of age-related chronic diseases such as stroke, heart disease, osteoporosis and Alzheimers. Remote health monitoring is one way to try to address the rising healthcare costs associated with caring for aged or chronically ill persons. In fact, the healthcare market is among the fastest growing markets for WiFi and other Wireless LAN Technologies [6]. Many wireless mobile devices have been developed to monitor people's health and wellbeing especially those suffering from diabetes, high blood pressure or heart disease. For example, the U.S Company Globus uses the Paradigm Diabetes Management System, a blood glucose monitor and insulin pump that work together to manage diabetes wirelessly [7]. Schwiebert et al [8] have described their challenges in wireless networking of human embedded smart sensor arrays in developing a retina prosthesis. [9]. In Australia, the company Pro Medicus, a secure smart messaging system for transmitting pathology results to over 19000 doctors, is proving a success story in IT Healthcare[10].

Our system provides for aged or ill persons who move back to their homes from hospital or care facilities yet still need health monitoring. The health care centre we envision can be compared to the e-Intensive Care Unit (ICU $®$ ) described by Visicu on their webpage [11] but tailored to 'smart home' care. The ICUß units have saved lives and reduced costs [12]. The 'smart home' care system can also be compared to HomMed [13] but can integrate more functionalities (fall detection, video, sleep disorders, heart attacks and problems with obesity). With our system, patients are free to move about in their own home and further a field, secure in the knowledge that they are being monitored. Just as airplanes are monitored by air traffic controllers, our patients will be monitored remotely by healthcare providers who will have access to the patient's information via a web server and, in the case of an emergency or at a predetermined time, via audio and video link up. We intend to integrate IP Webcams in our systems to provide this extra security dimension for clients who will feel confident if they know that video surveillance will automatically start in case of an emergency or if the client requires it as a specified time.

This paper describes a prototype we developed for remote healthcare monitoring. Section 2 presents various scenarios each describing a typical situation and illustrates how our prototype is used. Section 3 gives the status of our prototype and research work. Section 4 concludes this paper.

\section{SCENARIOS}

This section discusses several scenarios showing the daily use of our application from the perspective of a patient (Marie) and a health centre staff member (James). Marie has had a stroke and is about to leave the hospital. Marie's mobility has been severely affected by the stroke and, although she is able to walk, she is very concerned that she will fall and not be discovered quickly enough. To reduce her anxiety the stroke specialist offers Marie the use of a smart phone and sensors that will monitor her 24 hours a day. Stroke is a common cause of death and severe acquired disability. Stroke specialization includes elements of neurology, cardiovascular disease, ageing and rehabilitation. Additionally, a technician will install wireless webcams in Marie's house to monitor her in case of an emergency and interact with her at agreed times. 


\section{A. Seeing the Stroke Specialist}

The specialist performs several health checks to determine what parameters need to be monitored on a daily basis and the threshold levels for each sensor. The blood pressure is taken and reveals that Marie suffers from moderate hypertension. Her blood pressure needs to come down and the specialist prescribes a blood pressure-reducing medication. The specialist instructs Marie to measure her blood pressure in the morning and before going to bed at night. After weighing Marie, the specialist informs her she must lose some weight. The specialist puts her on a diet. The specialist also checks whether she has diabetes but the glucose levels are within normal limits.

The specialist is now ready to configure the tele-monitoring application directly on the smart phone. To detect a fall, Marie has to wear an accelerometer day and night. Figure 1 shows a 3 -axis accelerometer sensor which can be easily attached to the body. This is a non intrusive sensor that is wirelessly connected to a smart phone via Bluetooth.

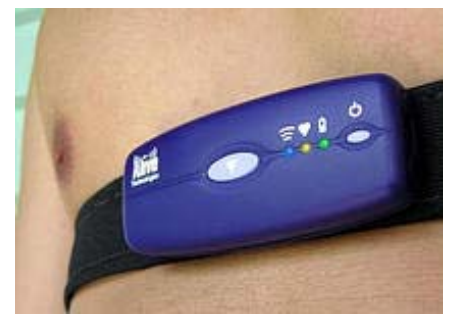

Figure 1. 3-Axis Accelerometer from Alive Technologies

Figure 2 illustrates how the specialist configures the accelerometer and weight sensor on the smart phone. The configuration settings are password protected so that only a specialist can configure the sensors. The accelerometer sensitivity can be adjusted to the person's movement characteristics.

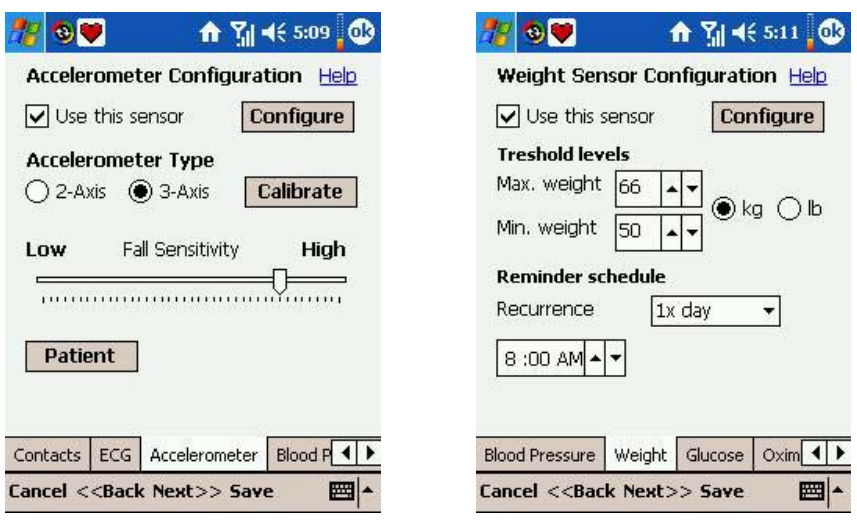

Figure 2. Patient specific configuration.

The specialist configures the weight sensor and sets the maximum and minimum weight for Marie. Marie has to weigh herself daily around 8 o'clock in the morning. The application will automatically start-up at 8am and notify Marie to take the weight measurement. For our system we used a Bluetooth enabled weight scale that transmits the weight automatically to the smart phone.
Next the specialist sets the threshold levels for the blood pressure monitor and instructs Marie how to measure her blood pressure correctly as depicted in Figure 3 below. The blood pressure monitor transmits the measurements automatically to the smart phone via Bluetooth.

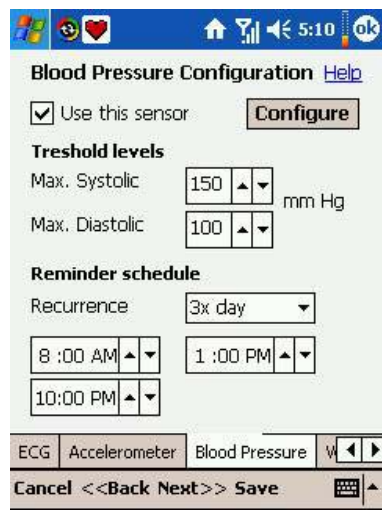

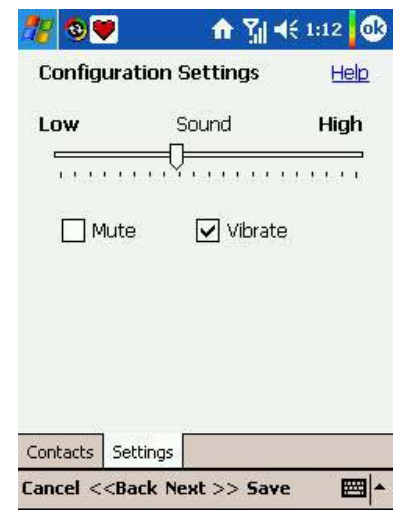

Figure 3. Patient specific configuration.

It is important that elderly and disabled people are not overwhelmed by the technology so Marie only needs to push one button on each device to have the values automatically transmitted to the smart phone.

The specialist also configures how the application will warn Marie. In this case Marie will be warned by sound and vibration when she has to take a measurement (figure 2, right). Marie will be able to change those settings when desired. Figure 4 illustrates the Weight Scale and the Blood pressure monitor that Marie will use.
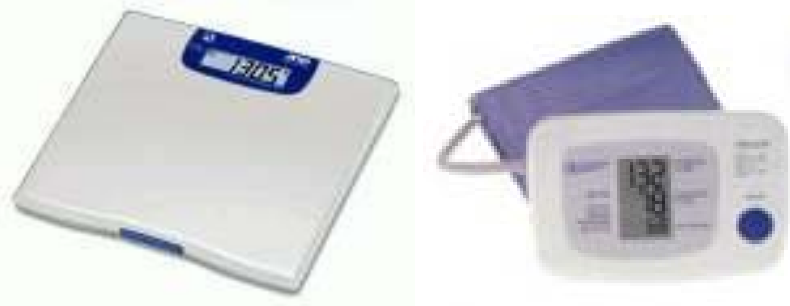

Figure 4. A\&D Bluetooth Weight Scale and Blood Pressure Monitor

A technician visits Marie at home and installs wireless webcams that will be used in case of an alarm (see Figure 5 below). The webcams [17] can be either wall mounted or ceiling mounted, or placed on a desktop. The webcams can be remotely controlled with pan/tilt control functions which adjust the camera angle. Additionally the webcams are equipped with two-way audio transmission which enables the person in the healthcare centre to communicate with Marie. The technician strategically positions the webcams so that Marie can be monitored everywhere in the house. It should be noted that the cameras will only be activated in case of an emergency situation or at agreed time. Marie will be notified by sound and the lights in the house will be turned on when the cameras are activated. It is important that Marie does not feel she is under constant surveillance as privacy in her home is of paramount importance. On the other hand, Marie is an elderly person who finds that visiting the doctor and the health centre can be a trial so the system has the flexibility to allow Marie to schedule 
video and audio interviews at convenient times with the physician or with James or other health staff members should she so desire.

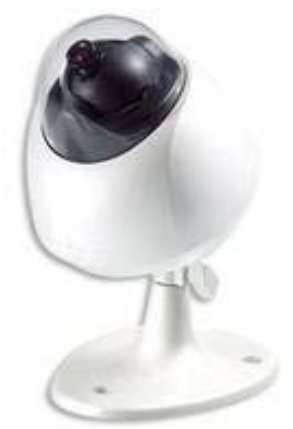

Figure 5. Wireless IP webcam with two-way audio

\section{B. A normal day for the patient in the house}

Marie has been at home for a few days and has familiarized herself with the medical monitoring devices that have been supplied to ensure her continued well being. Each day, she weighs herself on the weight scale and takes her blood pressure. The data is automatically sent to the smart phone which forwards it onto the web server where it may be viewed by the health centre personnel. As directed by the specialist Marie also keeps a log of her weight and blood pressure along with any incidents that could explain a change in the readings (e.g. negative stress: death of a friend). Keeping a $\log$ is achieved by a simple interface on the smart phone where Marie can select an activity from a dropdown menu. The benefit of a $\log$ is that it allows the specialist to understand what activities Marie has done which could have caused a change in the blood pressure reading or weight. She uses the smart phone the specialist lent to her as her everyday mobile phone.

It is vital that she keeps the sensor on her body around the clock as there is always a danger of falling when getting up to go to the bathroom. The smart phone stays in the cradle/charger overnight to charge the battery. As long as she stays within 20 meters of the smart phone she is monitored. Marie feels more secure in her home now that she is monitored 24/7.

When Marie places the smart phone in the cradle, which is connected via her PC to the Internet, the application automatically uploads the recorded data to the health centre database. The application records normal and non urgent warnings and can download new configuration data to the smart phone application as set by the specialist.

Some sensor threshold levels are set to congratulate or encourage Marie. For example, when Marie lost her first kilo today, the weight monitor application congratulated her. Sensor measurements that are dangerously out of range will be transmitted directly to the health centre via GPRS and not wait till the next synchronization. This allows for the specialist or nurse to react immediately. When a blood pressure reading is out of range compared to a previous reading, the application will instruct Marie to measure the blood pressure again to avoid incorrect readings.

\section{A normal day for the patient outside the house}

Even though Marie has had a stroke, the specialist advised her to exercise by going for a short walk everyday. The specialist configured the smart phone to be connected to a Bluetooth GPS receiver and Marie activates the GPS receiver when she is ready. The GPS and the application can track Marie while she is walking in the park and in clear sight of GPS satellites. This allows the ambulance to locate her in case Marie has a fall en route to the house.

\section{A normal day at the Healthcare centre}

In our system, a connection to the healthcare centre (aka ePersonalized Rehabilitation Monitoring System) is needed to deal with 'smart home' care situations. This healthcare centre monitors patients who have registered for their services. Some patients will only use a smart phone and a heart monitor application as described by Gay and Leijdekkers [14] while others use wireless web cameras and other sensing devices such as weight scales, accelerometers and blood pressure sensors. At the healthcare centre, staff members like James have access to their patients' records and, in case of an emergency, the patient needing attention appears on the main screen.

There is an emergency involving Marie and James is dealing with it. On his screen he has access to Marie's latest vital sign information, her medical history and her location. Having access to this information enables James to make fast decisions and send immediate help to the right location if necessary. In Marie's case, he suspects a fall and James can see the video images from the patient's house and therefore he is able to locate Marie and confirm the fall. James can also talk to Marie using the 2-way audio feature of the camera. She is still conscious but she feels a sharp pain in her leg. James reassures Marie, tells her not to move and the paramedics are sent to her house with enough information to deal with Marie's condition (for example, allergy to medication, type of fall and previous stroke).

When not dealing with an emergency, James deals with patients who have worrying, but not yet life-threatening, vital signs. Some patient will, for example, need to weigh themselves and measure their blood pressure every morning. If the readings are out of the range set by the specialist, James checks the history of that person to decide whether it is fine, whether he should call the patient and get some new vital sign readings or ask the patient to call the doctor.

For example, if James identifies a potentially dangerous blood pressure reading he can call a patient to get another blood pressure measurement. It might turn out that he realizes that the cuff is simply wrongly positioned but, if the readings stay out of range, he will make a decision and give medical advice to the patient.

Some patients will come to visit the healthcare centre to discuss any concerns they have about their health. It is also the opportunity to change some of the parameters of the monitoring devices to adapt to the progress/evolution of the patient's health. 


\section{SMART HOME AND PERSONALIZED HEALTH MONITORING PROTOTYPE}

The scenarios described above illustrate the potential use of the prototype we are developing at the University of Technology, Sydney.

Figure 6 depicts the architecture of the smart home care system. It consists of 2 parts namely the smart phone application and the healthcare centre server. The smart phone communicates with the home server via WiFi or cable. The home server runs software to control the webcams and controls the information exchange with the health care server via an ADSL connection.

The smart phone application is developed using Microsoft's Windows Mobile Pocket PC platform. We selected this platform due to easy access to lower level APIs which are needed for the sensor manager modules.
- a ECG/3-axis accelerometer sensor from Alive Technologies [18] to detect life threatening arrhythmias and falls.

- a Blood Pressure Monitor and Scale from A\&D Medical [19].

The design of the healthcare centre servers is based on Microsoft ASP.net. ASP.net is Microsoft's main proprietary technology for creating dynamic web applications. We use web services to facilitate the smart phone's communication to the remote healthcare server. The web services currently implemented allow the smart phone to upload weight scale readings, blood pressure readings as well as update alarm values on the Smart phone.

The home server uses .Net technology and runs a web service application to remotely control the webcams and light switches. The lights in the house are remotely controlled using

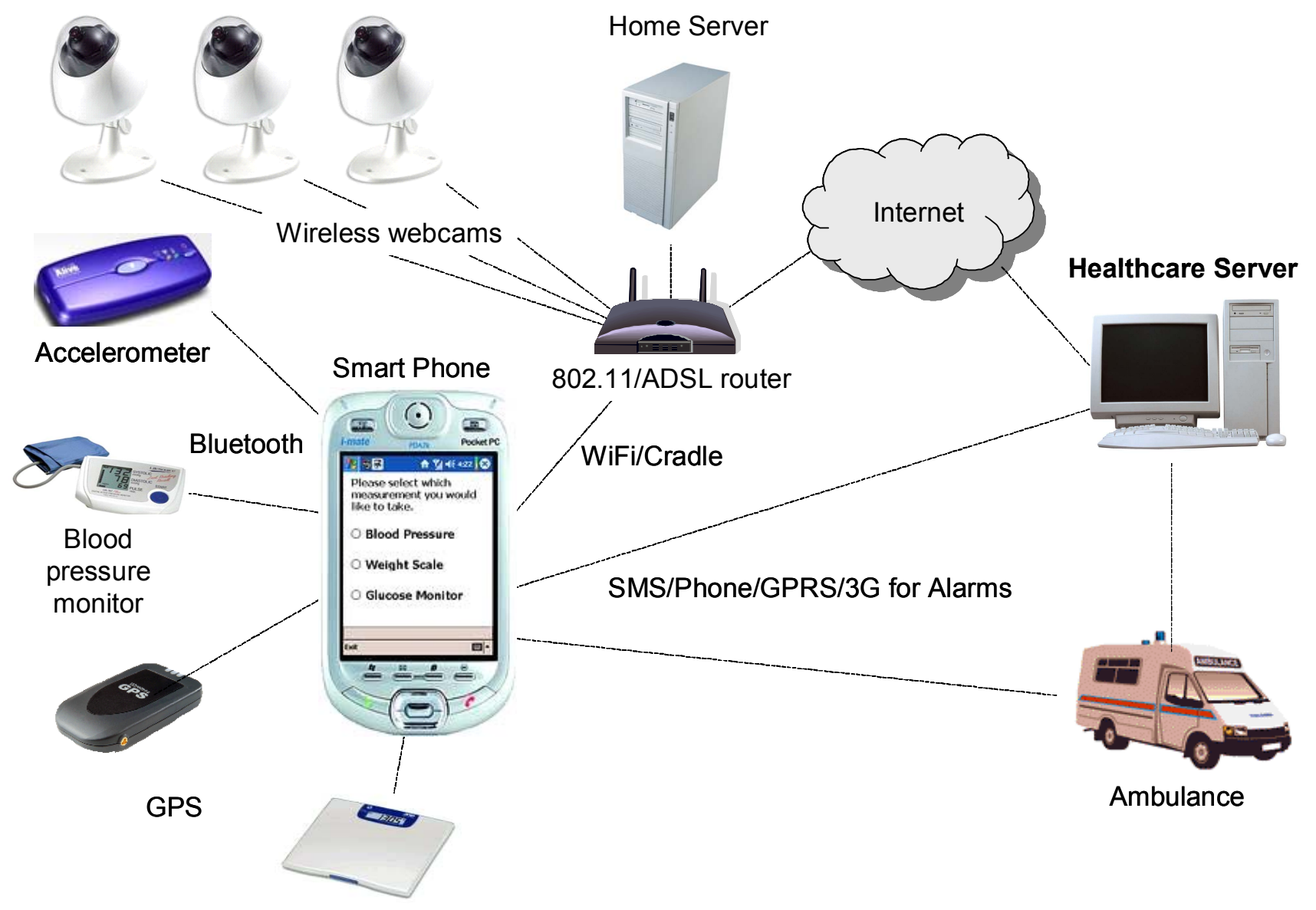

Figure 6 - Smart home and personalized health monitoring architecture

Also the tight integration with the operating system allows easier access to other applications running on the mobile device such as the calendar application, WiFi and the GSM cellid information. We used the .Net Compact Framework extended with OpenNETCF [20] modules to build the application. The data is stored in an SQL CE Server which is a compact database for mobile devices.

The Bluetooth enabled devices we are using are
X10 technology. The X10 protocol [16] uses the power line wiring to send control signals to X10 enabled switches.

\section{A. Accelerometer}

Accelerometers are widely used to monitor human body activity. We implemented an algorithm developed by Garret Brown [15]. This algorithm uses a state-machine that analyzes data from a 3-axis accelerometer worn on a waist belt. The algorithm focuses on large accelerations and the user's upper 
body position. After a large acceleration the user's position is analysed. A fall is detected when the user's position is horizontal or not upright after some period of inactivity. An acceleration is not classified as a fall when the position is upright or the accelerometer detects activity. Based on Brown's testing (123 falls and 36 non falls), the algorithm is able to detect around $90 \%$ of all the falls along with $5 \%$ of false positives. The use of cameras is a useful complement to this algorithm and can confirm the fall and eliminate false positives.

\section{CONCLUSION}

The exponential growth in the number of aged persons has increased the pressure on governments to provide remote monitoring for those who wish to remain at home whilst still being secure in the knowledge that medical help is just a 'click' away. In the system we developed, the elderly will be able to keep a check on their health status from home and whilst moving around. This will improve their quality of life as well as save governments from expending huge amounts of money on hospital and aged care facilities. Our system provides a 24/7 personalized health monitoring system specifically for people who are either prone to falling and/or who are overweight, using smart phones and wireless wearable sensors. It is simple to operate and non-threatening for elderly persons. Our personalized system enables analysis of the sensor data on a local or remote device in real-time and/or at a later time for trend analysis. It has inbuilt emergency and location functions which mean that external assistance can be called for automatically in the case of an emergency. The use of web cameras which provide both audio and video linkage provide an extra level of monitoring but it must be mentioned that such video surveillance could be criticized by some as an invasion of privacy. We have attempted to overcome this criticism by proposing that the video surveillance is only used at times of emergency or for scheduled meetings.

By processing the data locally on the smart phone, a patient is monitored and reassured without needing to be continuously connected to a healthcare centre. This reduces the workload of medical staff, communication costs and motivates the patient's self-care. The connection to a healthcare centre is important since there are limits to what can be processed locally and monitored automatically. We need the expertise of medical staff to complement our application.

Our future work will examine medical device standards and the reliability and security of Bluetooth when communicating health data. There is an explosion of proprietary devices that record medical data and, if the area of tele-health is to progress, these various devices must be able to interact and exchange compatible data. Bluetooth has addressed the compatibility issue but questions about its reliability and security, particularly in the health arena, still remain.

We also intend to pilot test our system with a control study to compare outcomes and patient perceptions of a monitored group and a non-monitored group. Once we have figures on the acceptability and utility of the system we will develop a larger randomized controlled trial which will test efficacy and effectiveness.

\section{REFERENCES}

[1] Dishman E. 2004, Inventing Wellness Systems for Aging in Place, IEEE Computer, May 2004, pp.34-41

[2] CDC, 2005, Falls and Hip Fractures among Older Adults, http://www.cdc.gov/ncipc/factsheets/falls.htm (viewed 5 September 2006).

[3] Murphy SL. Deaths: Final data for 1998. National Vital Statistics Reports, vol. 48, no.11. Hyattsville (MD): National Center for Health Statistics; 2000

[4] New England Journal of Medicine 2004, http://content.nejm.org

[5] Gururajan, R, Murugesan, S., \& Soar, J. 2005, 'Introducing Mobile Technology in Support of Healthcare', Cutter IT Journal the Journal of Information Technology Management, Vol.18, No.8, pp. 12-18.

[6] PBI Media, "Strong Prognosis for Wireless in Healthcare", Communications Today, February 24, 2003, http://www.findarticles.com/cf 0/m0BMD/35 9/98013124/p1/article.jht $\underline{\mathrm{ml}}$, (viewed 5 September 2006).

[7] "Managing Diabetes with Wireless Insulin Pump: Patients Input Diet Plan, Device Matches Insulin Levels" February 16, 2004 http://www.thewbalchannel.com/womansdoctor/2850769/detail.html (viewed 5 September 2006).

[8] D. Konstantas, "The Mobihealth Project. IST Project" IST-2001-36006, European Commission: Deliverable 2.6, http://www.mobihealth.org, 2004 (viewed 5 September 2006).

[9] H. Zhuge, "Exploring an Epidemic in an e-Science Environment," Communications of the ACM, September 2005, vol 48, no 9

[10] K. Dearne, "Project Flatline," Australian IT, 6 Sept 2005, p1.

[11] Visicu Webpage, http://www.visicu.com/ and videos http://www.visicu.com/press/mulitmedia.html (viewed 5 September 2006).

[12] Visicu pressroom, http://www.visicu.com/press/reporterresources/presskit.html (viewed 5 September 2006).

[13] HomMed Home page, http://www.hommed.com/ (viewed 5 September 2006).

[14] Valérie Gay, Peter Leijdekkers, 'Around the Clock Personalized Health Monitoring Using Smart Phones ', Smart Homes and Beyond ICOST2006 4th International Conference On Smart homes and health Telematics, Edited by Chris Nugent, Juan Carlos Augusto,ISBN 158603-623-8 pp 82 - 89, Belfast, UK - June 26-28, 2006

[15] Garret Brown. An accelerometer based fall detector: Development, experimentation, and analysis. 2005. University of California, Berkeley.

[16] Wikipedia, X10 definition (industry standard) http://en.wikipedia.org/wiki/X10 (viewed 5 September 2006).

[17] Tiglion Wireless IP Webcam, product description, http://tiglon.en.alibaba.com/product/50173283/50994096/IP Webcams/ Wireless IP Webcam.html (viewed 5 September 2006).

[18] Alive Technologies, http://www.alivetec.com/ (viewed 5 September 2006)

[19] A\&D Medical website http://www.andmedical.com.au/ (viewed 5 September 2006)

[20] OpenNETCF.org, 'The Premier .NET Compact Framework Shared Source Site' http://www.opennetcf.org (viewed 5 September 2006). 